\title{
W-band TWTs for New Generation High Capacity Wireless Networks
}

\author{
Claudio Paoloni \\ Rosa Letizia \\ Lancaster University \\ Lancaster, UK, LA1 4YW
Ralph Zimmerman
HFSE
Germany

\author{
Frédéric André \\ Sophie Kohler \\ Thales Electron Devices \\ SAS \\ Vélizy-Villacoublay, France \\ Viktor Krozer \\ Giacomo Ulisse \\ Goethe University of \\ Frankfurt \\ Frankfurt, Germany
}

\author{
François Magne \\ When-AB \\ France
}
Antonio Ramirez
Fibernova
Valencia, Spain

\author{
Marc Rocchi \\ Marc Marilier \\ OMMIC \\ Limeil-Brevannes, France \\ Ruth Vilar \\ Universitat Politecnica de \\ Valencia \\ Valencia, Spain
}

\begin{abstract}
The congestion of the spectrum actually devoted to wireless networks has stimulated the exploitation of the wide availability of frequencies in the millimeter wave range. The high atmospheric attenuation and the strong detrimental rain effect require level of power not available by solid state power amplifiers typically used at microwave frequency. The lack of power has so far limited the use of millimeter wave range.

The massive use of Traveling Wave Tubes as power amplifier in high capacity wireless networks will be the breakthrough in the development of the future millimeter wave network fundamental for the $5 G$ development. The H2020 TWEETHER project aims at a new wireless network concept based on TWTs to distribute data in the millimeter wave portion of the spectrum.
\end{abstract}

Keywords: Traveling wave tube, W-band, wireless networks, 5G, mobile, access, backhaul, linearity

\section{Introduction}

Conventional wireless networks are fed either by wireless point to point links or by fiber optics for the backhaul of base stations. However, the increasing request of data due to download and upload of a huge amount of videos and images is putting the actual network system under pressure.

The great excitement for the features of the future $5 \mathrm{G}$ and the need to provide broadband access in areas where fiber optics cannot be deployed are forcing operators and manufacturers toward the millimeter wave domain $[1,2]$.

The wide band frequency portions of the spectrum available above $70 \mathrm{GHz}$ permit multi-gigabit data rate. A number of wireless systems are already available in the Q, $\mathrm{V}$ and E-band. All these systems are point to point. This low power from solid state devices at millimeter waves only permits to generate very narrow beam for point to point links.

The breakthrough in the millimeter wave networks is in the distribution of data on a wide area as the actual wireless microwave networks provide.
The paper describes how the vacuum electronics will strongly change the paradigm of the future wireless networks for high data rate distribution.

\section{The scenario}

The future need of capacity is estimated in an average of $10 \mathrm{Gbps} / \mathrm{km}^{2}$. This is valid for access for residential fixed connections and for future small cell mobile networks.

This capacity is presently distributed by fiber or microwave links. However, fiber is expensive to deploy and is therefore mostly present in high density areas, while the suburban or rural regions are served with wireless microwave links of limited capacity.

The increase of capacity needs requires a new network concept providing improved cost effectiveness with regards to fiber optics and higher data rates than current point to point wireless solutions. The European project TWEETHER proposes a solution meeting both

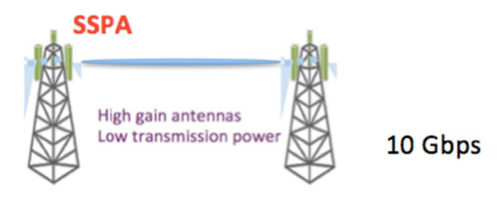

Actual point to point technology

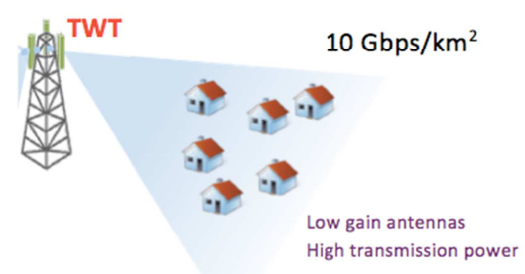

W-band TWT based wireless network

Fig. 1 Present and future high capacity wireless networks 
requirements: wireless point to multipoint networks at millimeter waves.

Currently, the available links at millimeter waves, due to the low power of solid state amplifiers, are Point to Point (Fig.1). These links are suitable for fiber replacement in some low capacity scenarios, but not for cost-effective access.

TWEETHER project assumes that a coverage for an area with a radius of $1 \mathrm{~km}$ could be considered a good balance in terms of TCO (total cost of operation), divided in 4 or more sectors, with $10 \mathrm{Gbps} / \mathrm{km}^{2}$ per sector, and a bandwidth need of $3 \mathrm{GHz}$. The power required for illuminating the area is estimated to be $40 \mathrm{~W}$ saturated.

No solid state device can provide this level of output power at millimeter waves. The most advanced GaN transistors could provide maximum $2 \mathrm{~W}$ saturated power with that bandwidth, much lower than needed [3].

\section{W-band TWT}

The W-band $(92-95 \mathrm{GHz})$ is a portion of the spectrum presently lightly regulated and suitable for high data rate. The attenuation and the lack of power amplifiers for the hub so far have made the use of these frequencies unfeasible.

The breakthrough in mm-waves wireless network is in the availability of coverage of an area to distribute capacity to a number of point of presences (users of fixed internet of small cells for $4 \mathrm{G}$ or $5 \mathrm{G}$ networks) (Fig.1).

A European project in the frame of the Horizon 2020 program is addressing the challenge.

The TWEETHER project "Traveling Wave Tube based W-band wireless network for high data rate, spectrum and energy efficiency" (www.tweether.eu) aims at a new way to distribute high capacity on large area by introducing for the first time a massive use of TWTs in the future high capacity wireless networks $[4,5]$. A purposely designed high power TWT can provide a signal level that will allow Point to multipoint distribution (Fig.1)

The project includes the design, fabrication and demonstration of TWTs for massive deployment in wireless hubs.

The TWT specifications are:

- Frequency: 92 - $95 \mathrm{GHz}$

- Gain: test TWT $30 \mathrm{~dB}$ minimum

- Output power: up to $40 \mathrm{~W}$

- Operating environment: outdoor $-10+55^{\circ}$

- Dimensions: less than $5 \mathrm{dm}^{3}\left(15 \mathrm{dm}^{3}\right.$ including the power supply)

- Weight: $<3 \mathrm{~kg}$

- Reliability: MTBF $>10^{6}$ hours

The design of all the part is in advanced status. In particular, a folded waveguide has been chosen for the

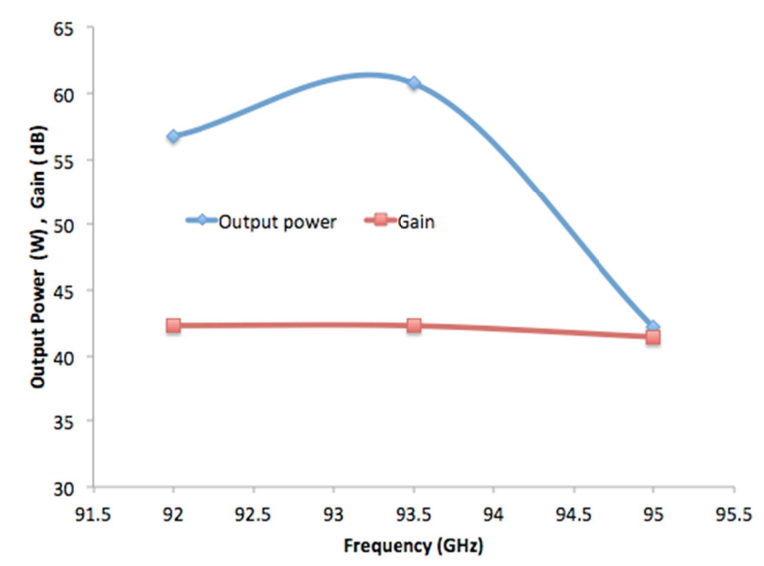

Fig.2 W-band TWT gain and output power

first TWT for the well known properties of wide band and relatively easy fabrication.

The preliminary performances are shown in Fig.2. The TWT was simulated by MAGIC3D. A gain above $40 \mathrm{~dB}$ and an output power above $40 \mathrm{~W}$ are obtained.

\section{Acknowledgements}

The project has received funding from the European Union's Horizon 2020 research and innovation program under grant agreement no 644678.

\section{References}

1. T.S. Rappaport et al., " Millimeter Wave Mobile Communications for 5G Cellular: It will work!”, IEEE Access, pp. 335-349, Mai 2013.

2. C. Colombo; M. Cirigliano "Next-generation access network: A wireless network using E-band Radio frequency (71-86 Ghz) to provide wideband connectivity," in Bell Labs Technical Journal, vol.16, no.1, pp.187-205, June 2011J.

3. Schellenberg, J.; Bumjin Kim; Phan, T., "W-Band, Broadband 2W GaN MMIC," in Microwave Symposium Digest (IMS), 2013 IEEE MTT-S International, 2-7 June 2013

4. C. Paoloni and M. Mineo, "Double Corrugated Waveguide for G-Band Traveling Wave Tubes,", IEEE Trans. on Electron Devices, vol.61, no.12, pp.4259,4263, Dec. 2014

5. M. Kotiranta, V. Krozer, V. Zhurbenko, "Square helix TWT for THz frequencies," 35th International Conference on Infrared, Millimetre, and Terahertz Waves, 2010 